Proceedings of the 15th Czech and Slovak Conference on Magnetism, Košice, Slovakia, June 17-21 2013

\title{
Minor Loop Scaling Rules for Finemet Type Soft Magnetic Cores
}

\author{
L.K. VARGA ${ }^{a, *}$, J. KOVÁC̆ ${ }^{b}$ \\ ${ }^{a}$ Wigner Research Center for Physics, Institute for Solid State Physics and Optics, Hungarian Academy of Sciences, \\ 1525 Budapest, P.O.B.49, Hungary \\ ${ }^{b}$ Institute of Experimental Physics SAS, Watsonova 47, 04001 Košice, Slovakia
}

\begin{abstract}
The validity of the Steinmetz law $\left(P=K B^{n}\right)$ has been analyzed using the tanh model, by splitting the minor loops in three or two components, corresponding to different magnetization processes at different excitation levels. It is shown that the original Steinmetz law with the exponent $n=1.6$ is valid only at and above the coercive field, for intermediate and high induction values, where all the three magnetization processes are present. At small magnetization levels the exponent is about 3, much higher than the original proposed value.
\end{abstract}

DOI: $10.12693 /$ APhysPolA.126.156

PACS: $75.50 . \mathrm{Bb}, 75.50 . \mathrm{Kj}, 75.60 .-\mathrm{d}, 75.60 . \mathrm{Ej}$

\section{Introduction}

Soon after the discovery of the DC magnetic hysteresis loss $P$ by Warburg in 1881 , formulated as $P=\oint H d B$, Steinmetz $[1,2]$ found a simple scaling expression of $P$ versus the magnetic induction $B$, as $P=k B^{n}$, where $k$ is a proportionality factor, depending on the material and $n=1.6$ is supposed to be unique for all soft magnetic materials. It is interesting, that so far there is no accepted explanation for this old law, although it is widely used in practical applications. Today we are facing a revived interest in this [3], and related [4, 5] scaling laws in magnetization processes.

The DC hysteresis loss has been decreased by more then two orders of magnitude during the last century, from $1100 \mathrm{~J} / \mathrm{m}^{3}$ at $1.5 \mathrm{~T}$ [2] for technically pure iron sheet, to $\sim 5 \mathrm{~J} / \mathrm{m}^{3}$ for the optimum annealed Finemet type nanocrystalline alloy. This is why it is of foremost importance to check the validity of the Steinmetz law over a large range of hysteresis losses. The order of magnitude of the DC hysteresis loop area can be estimated as $P \sim 4 H_{c}(B) B$, which compared to the Steinmetz law gives an estimated value of $H_{c}(B)=k^{\prime} B^{0.6}$. Therefore either the exponent of 1.6 for the hysteresis loss, or the coercivity exponent of 0.6 should be verified experimentally and modeled theoretically. The simple Steinmetz's power low was assumed to be valid for the entire magnetization region [6] but its validity has not been checked in the Rayleigh region, at low magnetization levels.

In this paper we investigate the validity of Steinmetz law from zero to saturation magnetization for the nanocrystalline Finemet type soft magnetic material having a round-type hysteresis loop obtained after normal heat treatment $\left(540^{\circ} \mathrm{C} / 1 \mathrm{~h}\right)$ of the amorphous precursor ribbon.

*corresponding author; e-mail: varga@szfki.hu

\section{Experiment}

A series of quasi DC hysteresis loops with varying excitation level have been measured by using the experimental arrangement shown in Fig. 1. Before the start of the measurement the toroidal sample was carefully demagnetized. The hysteresis loops were measured with a triangular excitation of $f=0.01 \mathrm{~Hz}$.

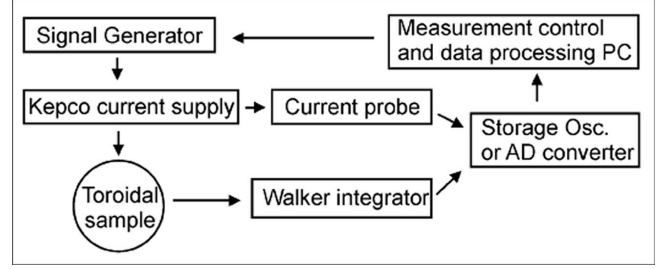

Fig. 1. Setup for hysteresis measurements at very low frequencies.

\section{Results and discussion}

A set of minor loops have been recorded for round (R) type Finemet toroidal samples. The minor loops are shown in the Fig. 2.only for brevity. The following parameters, such as the area, $P\left(B_{m}\right)$, the coercivity, $H_{c}\left(B_{m}\right)$ and the remanence,

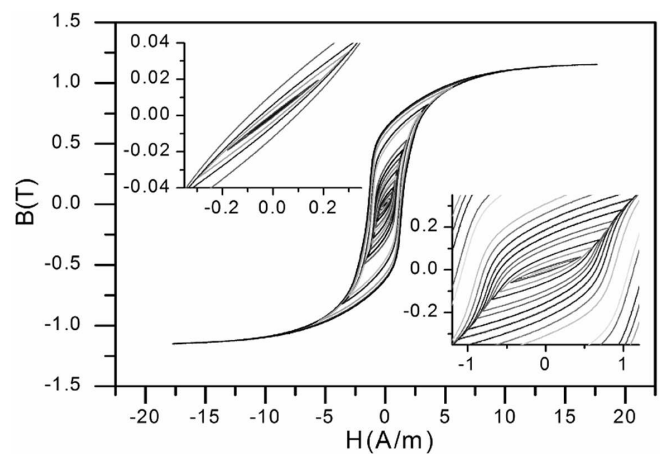

Fig. 2. Set of minor loops for Finemet sample nanocrystallized at $540^{\circ} \mathrm{C}$ for $1 \mathrm{~h}$ (Round type loop). 
The Rayleigh model of magnetization in small fields shows that the power law exponent near 3 is related to the reversible magnetization, whereas the exponent near 1.5 is linked to the irreversible magnetization process around and above the coercive field. Approximating the power loss as $P \sim 4 H_{m} B_{m}$, the corresponding exponents for $H_{c}$ versus $B_{m}$ dependences would be 2 and 0.5 , respectively, see Fig. 3.

The magnetization process in larger fields is supposed to consists of three overlapping processes: domain wall motion (DWM), domain wall annihilation and nucleation (DWAN) and domain rotation (DR). For details see [7].

The equations of the hyperbolic model in normalized form:

$$
\begin{gathered}
\frac{B}{B s}=A_{D W M} \tanh \left(\alpha_{D} \frac{H \pm H_{C, D W M}}{H s}\right)+ \\
A_{D W A N} \tanh \left(\alpha_{N} \frac{H \pm H_{C, D W A N}}{H s}\right)+ \\
A_{D R} \tanh \left(\alpha_{R} \frac{H \pm H_{C, D R}}{H s}\right)
\end{gathered}
$$

where $A_{D W M}+A_{D W A N}+A_{D R}=1$.

Figures $4 \mathrm{a}, 4 \mathrm{~b}$ and $4 \mathrm{c}$ show the loop components for minor loops, measured with maximal field $H_{m}$ well above, around, and below the $H_{c}$, respectively. The three components around and above $H_{c}$ follow the Steinmetz exponent, whereas the remaining two components below the $H_{c}$ have an exponent around 3 (see Fig. 5), in accordance with experimental findings.

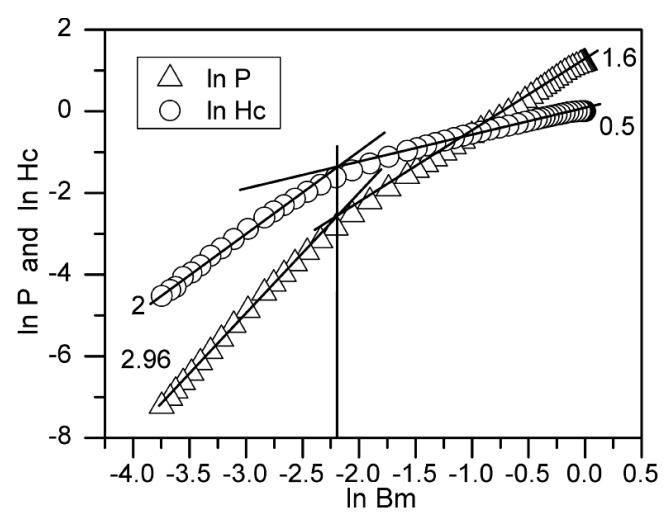

Fig. 5. The Steinmetz power low applied to experimental data and to the loop components, obtained by splitting the minor loops with the help of tanh model. Please observe that the DWAN component appears at large excitations only, whereas the DWM and DR components are present down to the lowest excitation available.

\section{Conclusions}

Splitting the minor loops in theoretical DWM, DWAN and DR components, using tanh model, helped us to interpret the larger Steinmetz exponent in Rayleigh region.

\section{Acknowledgments}

This work was supported by the Hungarian project OTKA 109570 and Slovakian projects VEGA 1/0861/12, 2-0192-13 and APVV-0492-11.

\section{References}

[1] C.P. Steinmetz, The Electrician January 2, 261 (1891).

[2] C.P. Steinmetz, Proceedings of the IEEE 72, 196 (1984), reprinted from American Institute of Electrical Engineers Transactions 9, 3 (1892).

[3] F.J.G. Landgraf, M. Emura, M.F. de Campos, J. Magn. Magn. Mater. 320, e531 (2008).

[4] S. Takahashi and L. Zhang, J. Phys.Soc. Japan 73, 1567 (2004).

[5] S. Takahashi, S. Kobayashi and T. Shishido, J. Phys.: Condens.Matter 20, 035217 (2008).

[6] R.M. Bozorth, Ferromagnetism, Van Nostrand, N.Y., 1951, p. 782.

[7] L.K. Varga, Gy. Kovács and J. Takács, J. Magn. Magn. Mater. 320, L26 (2008). 